\title{
A supplement to the convergence rate in a theorem of Heyde
}

\author{
Jianjun $\mathrm{He}^{*}$ and Tingfan Xie
}

"Correspondence: hejj@cjlu.edu.cn Department of Mathematics, China Jiliang University, Hangzhou, 310018, China

\begin{abstract}
Let $\left\{X, X_{n}, n \geq 1\right\}$ be a sequence of i.i.d. random variables with zero mean, set $S_{n}=\sum_{k=1}^{n} X_{k}, E X^{2}=\sigma^{2}>0$, and $\lambda(\epsilon)=\sum_{n=1}^{\infty} P\left(\left|S_{n}\right| \geq n \epsilon\right)$. In this paper, the authors discuss the rate of approximation of $\sigma^{2}$ by $\epsilon^{2} \lambda(\epsilon)$ under suitable conditions, improve the results of Klesov (Theory Probab. Math. Stat. 49:83-87, 1994), and extend the work He and Xie (Acta Math. Appl. Sin. 2012, doi:10.1007/s10255-012-0138-6).

MSC: $60 \mathrm{~F} 15 ; 60 \mathrm{G} 50$

Keywords: convergence rate; i.i.d. random variable; theorem of Heyde
\end{abstract}

\section{Introduction and main results}

Let $\left\{X, X_{n}, n \geq 1\right\}$ be a sequence of i.i.d. random variables, set $S_{n}=\sum_{k=1}^{n} X_{k}$, and $\lambda(\epsilon)=$ $\sum_{n=1}^{\infty} P\left(\left|S_{n}\right| \geq n \epsilon\right)$. Heyde [1] proved that

$$
\lim _{\epsilon \rightarrow 0} \epsilon^{2} \lambda(\epsilon)=\sigma^{2},
$$

whenever $E X^{2}=\sigma^{2}<\infty$ and $E X=0$.

There are various extensions of this result: Chen [2], Gut and Spătara [3], Lanzinger and Stadtmüller [4]. Liu and Lin [5] introduced a new kind of complete moment convergence; Klesov [6] studied the rate of approximation of $\sigma^{2}$ by $\epsilon^{2} \lambda(\epsilon)$ and proved the following Theorem A.

Theorem A Let $\left\{X, X_{n}, n \geq 1\right\}$ be a sequence of i.i.d. random variables with zero mean, if $E X^{2}=\sigma^{2}>0$, and $E|X|^{3}<\infty$, then

$$
\epsilon^{2} \lambda(\epsilon)-\sigma^{2}=o\left(\epsilon^{1 / 2}\right), \quad \text { as } \epsilon \rightarrow 0
$$

Recently, He and Xie [7] obtained Theorem B which improved Theorem A. Gut and Steinebach [8] extended the results of Klesov [6].

Theorem B Let $\left\{X, X_{n}, n \geq 1\right\}$ be a sequence of i.i.d. random variables, and $0<\delta \leq 1$, if

$$
E X=0, \quad E X^{2}=\sigma^{2}>0 \text { and } E|X|^{2+\delta}<\infty
$$

(c) $2012 \mathrm{He}$ and Xie; licensee Springer. This is an Open Access article distributed under the terms of the Creative Commons Attribution License (http://creativecommons.org/licenses/by/2.0), which permits unrestricted use, distribution, and reproduction in any medium, provided the original work is properly cited. 
then

$$
\epsilon^{2} \lambda(\epsilon)-\sigma^{2}= \begin{cases}O(\epsilon), & \delta=1 \\ o\left(\epsilon^{\delta}\right), & 0<\delta<1\end{cases}
$$

Let $G$ be the set of functions $g(x)$ that are defined for all real $x$ and satisfy the following conditions: (a) $g(x)$ is nonnegative, even, nondecreasing in the interval $x>0$, and $g(x) \neq 0$ for $x \neq 0$; (b) $\frac{x}{g(x)}$ is nondecreasing in the interval $x>0$.

Let $G_{0}$ be the set of functions $g(x) \in G$ satisfying the supplementary condition (c) $\lim _{x \rightarrow \infty} \frac{g\left(x^{2}\right)}{x g(x)}=0$. Obviously, the function $g(x)=|x|^{\delta}$ with $0<\delta<1$ belongs to $G_{0}$ and does not belong to $G_{0}$ if $\delta=1$. The purpose of this paper is to generalize Theorem $\mathrm{B}$ to the case where the condition $E|X|^{2+\delta}<\infty$ is replaced by a more general condition $E|X|^{2} g(X)<\infty$ in which the function $g$ belongs to some subset of $G$. Denote $T_{g}(v)=E X^{2} g(X) I(|X|>v)$, $T_{g}(v)$ is a nonnegative nonincreasing function in the interval $v>0$, and $\lim _{v \rightarrow \infty} T_{g}(v)=0$ with $E X^{2} g(X)<\infty$. Now we state our results as follows.

Theorem 1.1 Let $\left\{X, X_{n} ; n \geq 1\right\}$ be a sequence of i.i.d. random variables with zero mean and $E X^{2}=\sigma^{2}>0$, if $E X^{2} g(X)<\infty$ for some function $g(x) \in G$, and

$$
\sum_{n=1}^{\infty} \frac{1}{n g(\sqrt{n})}<\infty
$$

then

$$
\epsilon^{2} \lambda(\epsilon)-\sigma^{2}=O\left(\epsilon^{1 / 2}\right)+o(1)\left(h_{1}(\epsilon)+f_{1}(\epsilon)\right), \quad \text { as } \epsilon \rightarrow 0,
$$

where $f_{1}(\epsilon)=\sum_{n=\left[\frac{1}{\epsilon^{2}}\right]+1}^{\infty} \frac{1}{n g(\sqrt{n})}, h_{1}(\epsilon)=\epsilon^{2} \sum_{n=1}^{\left[\frac{1}{\epsilon^{2}}\right]} \frac{1}{g(\sqrt{n})}$.

Theorem 1.2 Under the conditions of Theorem 1.1, and $g(x) \in G_{0}$, then

$$
\epsilon^{2} \lambda(\epsilon)-\sigma^{2}=o(1)\left(h_{1}(\epsilon)+f_{1}(\epsilon)\right), \quad \text { as } \epsilon \rightarrow 0 .
$$

Throughout this paper, we suppose that $\mathrm{C}$ denotes a constant which only depends on some given numbers and may be different at each appearance, and that $[x]$ denotes the integer part of $x$.

\section{Proofs of the main results}

Before we prove the main results we state some lemmas. Lemma 2.1 is from [7]. $\Phi(x)$ is the standard normal distribution function, $\Phi(x)=\frac{1}{\sqrt{2 \pi}} \int_{-\infty}^{x} e^{-t^{2} / 2} d t$.

Lemma 2.1 Let $\left\{X, X_{n}, n \geq 1\right\}$ be a sequence of i.i.d. standard normal distribution random variables. Then

$$
\epsilon^{2} \lambda(\epsilon)=\epsilon^{2} \sum_{n=1}^{\infty} \frac{2}{\sqrt{2 \pi}} \int_{\epsilon \sqrt{n}}^{\infty} e^{-t^{2} / 2} d t=1-\frac{\epsilon^{2}}{2}+O\left(\epsilon^{3}\right), \quad \text { as } \epsilon \rightarrow 0 .
$$


If $\left\{X_{n}, n \geq 1\right\}$ is a sequence of independent random variables with zero mean and finite variance, and put $E X_{j}^{2}=\sigma_{j}^{2}, B_{n}=\sum_{j=1}^{n} \sigma_{j}^{2}$, Bikelis [9] obtained the following inequality:

$$
\begin{aligned}
\mid P( & \left.\frac{1}{\sqrt{B_{n}}} \sum_{j=1}^{n} X_{j}<x\right)-\Phi(x) \mid \\
\leq & C\left\{B_{n}^{-1}(1+|x|)^{-2} \sum_{j=1}^{n} \int_{|u|>(1+|x|) B_{n}^{1 / 2}} u^{2} d V_{j}(u)\right. \\
& \left.+B_{n}^{-3 / 2}(1+|x|)^{-3} \sum_{j=1}^{n} \int_{|u| \leq(1+|x|) B_{n}^{1 / 2}}|u|^{3} d V_{j}(u)\right\},
\end{aligned}
$$

for every $x$, where $V_{j}(x)=P\left(X_{j}<x\right)$ is the distribution function of the random variable $X_{j}$. $B y$ applying the above inequality to the sequence of i.i.d. random variables with zero mean and variance 1, and letting $|x|=\epsilon \sqrt{n}$, we have the following lemma.

Lemma 2.2 Let $\left\{X, X_{n}, n \geq 1\right\}$ be a sequence of i.i.d. random variables with zero mean and $E X^{2}=1$. Then for any given $\epsilon>0$, we have

$$
\begin{aligned}
& \left|P\left(\left|S_{n}\right|>n \epsilon\right)-\frac{2}{\sqrt{2 \pi}} \int_{\epsilon \sqrt{n}}^{\infty} e^{-t^{2} / 2} d t\right| \\
& \leq C(1+\epsilon \sqrt{n})^{-2} \int_{|u|>(1+\epsilon \sqrt{n}) \sqrt{n}} u^{2} d V(u) \\
& \quad+C^{-1 / 2}(1+\epsilon \sqrt{n})^{-3} \int_{|u| \leq(1+\epsilon \sqrt{n}) \sqrt{n}}|u|^{3} d V(u),
\end{aligned}
$$

where $V(x)=P(X<x)$ is the distribution function of a random variable $X$.

Proof of Theorem 1.1 Without loss of generality, we suppose that $\sigma^{2}=1,0<\epsilon<1$, and write

$$
\epsilon^{2} \lambda(\epsilon)=I+\epsilon^{2} \sum_{n=1}^{\infty} \frac{2}{\sqrt{2 \pi}} \int_{\epsilon \sqrt{n}}^{\infty} e^{-t^{2} / 2} d t
$$

where

$$
I=\epsilon^{2} \sum_{n=1}^{\infty}\left(P\left(\left|S_{n}\right|>n \epsilon\right)-\frac{2}{\sqrt{2 \pi}} \int_{\epsilon \sqrt{n}}^{\infty} e^{-t^{2} / 2} d t\right)
$$

Applying Lemma 2.1, we obtain

$$
\epsilon^{2} \lambda(\epsilon)=I+1-\frac{\epsilon^{2}}{2}+O\left(\epsilon^{3}\right)
$$

then

$$
\epsilon^{2} \lambda(\epsilon)-1=-\frac{\epsilon^{2}}{2}+\epsilon^{2} \sum_{n=1}^{\infty} R_{n}+O\left(\epsilon^{3}\right)
$$


here $R_{n}=P\left(\left|S_{n}\right|>n \epsilon\right)-\frac{2}{\sqrt{2 \pi}} \int_{\epsilon \sqrt{n}}^{\infty} e^{-t^{2} / 2} d t$. By Lemma 2.2,

$$
\left|R_{n}\right| \leq R_{1 n}+R_{2 n}
$$

where

$$
\begin{aligned}
& R_{1 n}=C(1+\epsilon \sqrt{n})^{-2} \int_{|u|>(1+\epsilon \sqrt{n}) \sqrt{n}} u^{2} d V(u), \\
& R_{2 n}=C n^{-1 / 2}(1+\epsilon \sqrt{n})^{-3} \int_{|u| \leq(1+\epsilon \sqrt{n}) \sqrt{n}}|u|^{3} d V(u) .
\end{aligned}
$$

We obtain

$$
\epsilon^{2} \lambda(\epsilon)-1=\epsilon^{2} \sum_{n=1}^{\infty} R_{1 n}+\epsilon^{2} \sum_{n=1}^{\infty} R_{2 n}+O\left(\epsilon^{2}\right) .
$$

Firstly, we estimate $\epsilon^{2} \sum_{n=1}^{\infty} R_{1 n}$. Note that

$$
\epsilon^{2} \sum_{n=1}^{\infty} R_{1 n}=\epsilon^{2} \sum_{n=1}^{\left[\frac{1}{\epsilon^{2}}\right]} R_{1 n}+\epsilon^{2} \sum_{n=\left[\frac{1}{\epsilon^{2}}\right]+1}^{\infty} R_{1 n}=: T_{1}+T_{2}
$$

Applying the condition $E X^{2} g(X)<\infty$, we have

$$
\lim _{n \rightarrow \infty} \int_{|u|>\sqrt[4]{n}} u^{2} g(u) d V(u)=0 .
$$

Therefore, for any $\eta>0$, there is an integer $N_{0}$ such that $\int_{|u|>\sqrt[4]{n}} u^{2} g(u) d V(u) \leq \eta$, whenever $n>N_{0}$. Hence

$$
\begin{aligned}
T_{1} & \leq C \epsilon^{2} \sum_{n=1}^{N_{0}} \int_{|u|>\sqrt{n}} u^{2} d V(u)+C \epsilon^{2} \sum_{n=N_{0}+1}^{\left[\frac{1}{\left.\epsilon^{2}\right]}\right.}(1+\epsilon \sqrt{n})^{-2} \int_{|u|>(1+\epsilon \sqrt{n}) \sqrt{n}} u^{2} d V(u) \\
& \leq C \epsilon^{2} N_{0}+C \epsilon^{2} \eta \sum_{n=N_{0}+1}^{\left[\frac{1}{\left.\epsilon^{2}\right]}\right.} \frac{1}{(1+\epsilon \sqrt{n})^{2} g(\sqrt{n}(1+\epsilon \sqrt{n}))} \\
& \leq C \epsilon^{2}\left(N_{0}+\eta \sum_{n=1}^{\left[\frac{1}{\epsilon^{2}}\right]} \frac{1}{g(\sqrt{n})}\right) \\
& =C h_{1}(\epsilon)\left(\frac{N_{0}}{\sum_{n=1}^{\left[\frac{1}{\left.\epsilon^{2}\right]}\right.} \frac{1}{g(\sqrt{n})}}+\eta\right) \\
& \leq C h_{1}(\epsilon)\left(N_{0} \epsilon+\eta\right) \\
& =o\left(h_{1}(\epsilon)\right),
\end{aligned}
$$


where $h_{1}(\epsilon)=\epsilon^{2} \sum_{n=1}^{\left[\frac{1}{\epsilon^{2}}\right]} \frac{1}{g(\sqrt{n})}$. For $T_{2}$, noting that $g(x) \in G$, we have the following inequality:

$$
\begin{aligned}
T_{2} & \leq C \epsilon^{2} \sum_{n=\left[\frac{1}{\epsilon^{2}}\right]+1}^{\infty} \frac{1}{n \epsilon^{2}} \int_{|u|>\sqrt{n}(1+\epsilon \sqrt{n})} u^{2} d V(u) \\
& \leq C \sum_{n=\left[\frac{1}{\epsilon^{2}}\right]+1}^{\infty} \frac{1}{n g(\sqrt{n}(1+\epsilon \sqrt{n}))} \int_{|u|>\sqrt{n}(1+\epsilon \sqrt{n})} u^{2} g(u) d V(u) \\
& \leq C \sum_{n=\left[\frac{1}{\epsilon^{2}}\right]+1}^{\infty} \frac{1}{n g(\sqrt{n})} \int_{|u|>\frac{1}{\epsilon}} u^{2} g(u) d V(u) \\
& \leq C T_{g}\left(\frac{1}{\epsilon}\right) f_{1}(\epsilon) .
\end{aligned}
$$

Next, we estimate the second term of (2.2). Note that

$$
\begin{aligned}
\epsilon^{2} \sum_{n=1}^{\infty} R_{2 n}= & C \epsilon^{2} \sum_{n=1}^{\infty} n^{-1 / 2}(1+\epsilon \sqrt{n})^{-3} \int_{|u| \leq(\sqrt{n}(1+\epsilon \sqrt{n}))^{1 / 2}}|u|^{3} d V(u) \\
& +C \epsilon^{2} \sum_{n=1}^{\infty} n^{-1 / 2}(1+\epsilon \sqrt{n})^{-3} \int_{(\sqrt{n}(1+\epsilon \sqrt{n}))^{1 / 2}<|u|<\sqrt{n}(1+\epsilon \sqrt{n})}|u|^{3} d V(u) \\
=: & J_{1}+J_{2} .
\end{aligned}
$$

For $J_{1}$, we can write

$$
\begin{aligned}
J_{1} & =C \epsilon^{2}\left(\sum_{n=1}^{\left[\frac{1}{\epsilon^{2}}\right]}+\sum_{n=\left[\frac{1}{\epsilon^{2}}\right]+1}^{\infty}\right) n^{-1 / 2}(1+\epsilon \sqrt{n})^{-3} \int_{|u| \leq(\sqrt{n}(1+\epsilon \sqrt{n}))^{1 / 2}}|u|^{3} d V(u) \\
& =: J_{11}+J_{12} .
\end{aligned}
$$

Noting that $\frac{x}{g(x)}$ is nondecreasing in the interval $x>0$, we have

$$
\begin{aligned}
J_{11} & =C \epsilon^{2} \sum_{n=1}^{\left[\frac{1}{\epsilon^{2}}\right]} \frac{1}{\sqrt{n}(1+\epsilon \sqrt{n})^{3}} \int_{|u| \leq(\sqrt{n}(1+\epsilon \sqrt{n}))^{1 / 2}}|u|^{3} d V(u) \\
& \leq C \epsilon^{2} \sum_{n=1}^{\left[\frac{1}{\epsilon^{2}}\right]} \frac{1}{n^{1 / 4}(1+\epsilon \sqrt{n})^{5 / 2} g\left((\sqrt{n}(1+\epsilon \sqrt{n}))^{1 / 2}\right)} \int_{|u| \leq(\sqrt{n}(1+\epsilon \sqrt{n}))^{1 / 2}} u^{2} g(u) d V(u) \\
& \leq C \epsilon^{2} \sum_{n=1}^{\left[\frac{1}{\epsilon^{2}}\right]} \frac{1}{n^{1 / 4} g\left(n^{1 / 4}\right)} \\
& =C h_{2}(\epsilon),
\end{aligned}
$$

where $h_{2}(\epsilon)=\epsilon^{2} \sum_{n=1}^{\left[\frac{1}{\epsilon^{2}}\right]} \frac{1}{n^{1 / 4} g\left(n^{1 / 4}\right)}$. 
Similarly, we can obtain

$$
\begin{aligned}
J_{12} & =C \epsilon^{2} \sum_{n=\left[\frac{1}{\epsilon^{2}}\right]+1}^{\infty} \frac{1}{\sqrt{n}(1+\epsilon \sqrt{n})^{3}} \int_{|u| \leq(\sqrt{n}(1+\epsilon \sqrt{n}))^{1 / 2}}|u|^{3} d V(u) \\
& \leq C \epsilon^{2} \sum_{n=\left[\frac{1}{\epsilon^{2}}\right]+1}^{\infty} \frac{1}{n^{1 / 4}(1+\epsilon \sqrt{n})^{5 / 2} g\left((\sqrt{n}(1+\epsilon \sqrt{n}))^{1 / 2}\right)} \int_{|u| \leq(\sqrt{n}(1+\epsilon \sqrt{n}))^{1 / 2}} u^{2} g(u) d V(u) \\
& \leq C \epsilon^{2} \sum_{n=\left[\frac{1}{\epsilon^{2}}\right]+1}^{\infty} \frac{1}{\epsilon^{5 / 2} n^{3 / 2} g\left(n^{1 / 4}\right)} \\
& =C \frac{1}{\sqrt{\epsilon}} f_{2}(\epsilon),
\end{aligned}
$$

where $f_{2}(\epsilon)=\sum_{n=\left[\frac{1}{\epsilon^{2}}\right]+1}^{\infty} \frac{1}{n^{3 / 2} g\left(n^{1 / 4}\right)}$.

For $J_{2}$, we write

$$
\begin{aligned}
J_{2} & =C \epsilon^{2}\left(\sum_{n=1}^{\left[\frac{1}{\epsilon^{2}}\right]}+\sum_{n=\left[\frac{1}{\epsilon^{2}}\right]+1}^{\infty}\right) n^{-1 / 2}(1+\epsilon \sqrt{n})^{-3} \int_{(\sqrt{n}(1+\epsilon \sqrt{n}))^{1 / 2}<|u|<\sqrt{n}(1+\epsilon \sqrt{n})}|u|^{3} d V(u) \\
& =: J_{21}+J_{22} .
\end{aligned}
$$

Using the properties of $g(x)$ by simple calculation, it follows that

$$
\begin{aligned}
J_{21}= & C \epsilon^{2} \sum_{n=1}^{\left[\frac{1}{\epsilon^{2}}\right]} n^{-1 / 2}(1+\epsilon \sqrt{n})^{-3} \int_{(\sqrt{n}(1+\epsilon \sqrt{n}))^{1 / 2}<|u|<\sqrt{n}(1+\epsilon \sqrt{n})}|u|^{3} d V(u) \\
\leq & C \epsilon^{2}\left(\sum_{n=1}^{N_{0}}+\sum_{n=N_{0}+1}^{\left[\frac{1}{\epsilon^{2}}\right]}\right) \frac{1}{(1+\epsilon \sqrt{n})^{2} g(\sqrt{n}(1+\epsilon \sqrt{n}))} \\
& \times \int_{(\sqrt{n}(1+\epsilon \sqrt{n}))^{1 / 2}<|u|<\sqrt{n}(1+\epsilon \sqrt{n})} u^{2} g(u) d V(u) \\
\leq & C \epsilon^{2}\left(\sum_{n=1}^{N_{0}}+\sum_{n=N_{0}+1}^{\left[\frac{1}{\epsilon^{2}}\right]}\right) \frac{1}{g(\sqrt{n})} \int_{|u|>n^{1 / 4}} u^{2} g(u) d V(u) \\
\leq & C \epsilon^{2}\left(N_{0}+\eta \sum_{n=1}^{\left[\frac{1}{\epsilon^{2}}\right]} \frac{1}{g(\sqrt{n})}\right) \\
= & o\left(h_{1}(\epsilon)\right),
\end{aligned}
$$

and

$$
\begin{aligned}
J_{22} & \leq C \epsilon^{2} \sum_{n=\left[\frac{1}{\epsilon^{2}}\right]+1}^{\infty} n^{-\frac{1}{2}}(1+\epsilon \sqrt{n})^{-3} \int_{(\sqrt{n}(1+\epsilon \sqrt{n}))^{1 / 2}<|u|<\sqrt{n}(1+\epsilon \sqrt{n})}|u|^{3} d V(u) \\
& \leq C \sum_{n=\left[\frac{1}{\epsilon^{2}}\right]+1}^{\infty} \frac{1}{n g(\sqrt{n})} \int_{\left(\sqrt{n}(1+\epsilon \sqrt{n})^{1 / 2}<|u|<\sqrt{n}(1+\epsilon \sqrt{n})\right.} u^{2} g(u) d V(u)
\end{aligned}
$$




$$
\begin{aligned}
& \leq C T_{g}\left(\frac{1}{\sqrt{\epsilon}}\right) \sum_{n=\left[\frac{1}{\epsilon^{2}}\right]+1}^{\infty} \frac{1}{n g(\sqrt{n})} \\
& \leq C T_{g}\left(\frac{1}{\sqrt{\epsilon}}\right) f_{1}(\epsilon) .
\end{aligned}
$$

From (2.2) to (2.8), we conclude that

$$
\epsilon^{2} \lambda(\epsilon)-1 \leq C \frac{1}{\sqrt{\epsilon}} f_{2}(\epsilon)+C T_{g}\left(\frac{1}{\sqrt{\epsilon}}\right) f_{1}(\epsilon)+o(1) h_{1}(\epsilon)+C h_{2}(\epsilon) .
$$

Since

$$
\frac{1}{\sqrt{\epsilon}} f_{2}(\epsilon) \leq \frac{C}{\sqrt{\epsilon}} \sum_{n=\left[\frac{1}{\epsilon^{2}}\right]+1}^{\infty} \frac{1}{n^{3 / 2}} \leq C \sqrt{\epsilon},
$$

and

$$
h_{2}(\epsilon)=\epsilon^{2} \sum_{n=1}^{\left[\frac{1}{\epsilon^{2}}\right]} \frac{1}{\sqrt[4]{n} g(\sqrt[4]{n})} \leq C \epsilon^{2} \sum_{n=1}^{\left[\frac{1}{\epsilon^{2}}\right]} \frac{1}{\sqrt[4]{n}} \leq C \sqrt{\epsilon},
$$

by (2.9), we have

$$
\epsilon^{2} \lambda(\epsilon)-1=O\left(\epsilon^{1 / 2}\right)+o(1)\left(f_{1}(\epsilon)+h_{1}(\epsilon)\right) .
$$

This completes the proof of Theorem 1.1.

Proof of Theorem 1.2 By the conditions $g(x) \in G_{0}$, and $\lim _{x \rightarrow \infty} \frac{g\left(x^{2}\right)}{x g(x)}=0$, for any $\eta>0$, there is an integer $N_{1}$ such that $\frac{g(\sqrt{n})}{\sqrt[4]{n} g(\sqrt[4]{n})} \leq \eta$, whenever $n>N_{1}$. We have

$$
\begin{aligned}
h_{2}(\epsilon) & \leq \epsilon^{2} \sum_{n=1}^{N_{1}} \frac{1}{\sqrt[4]{n} g(\sqrt[4]{n})}+\epsilon^{2} \sum_{n=N_{1}}^{\left[\frac{1}{\epsilon^{2}}\right]} \frac{\eta}{g(\sqrt{n})} \\
& \leq C \epsilon^{2} N_{1}+\epsilon^{2} \sum_{n=N_{1}+1}^{\left[\frac{1}{\epsilon^{2}}\right]} \frac{\eta}{g(\sqrt{n})} \\
& \leq C \epsilon^{2} N_{1}+\epsilon^{2} \sum_{n=1}^{\left[\frac{1}{\epsilon^{2}}\right]} \frac{\eta}{g(\sqrt{n})} \\
& =o(1) h_{1}(\epsilon),
\end{aligned}
$$

and

$$
\begin{aligned}
\frac{1}{\sqrt{\epsilon}} f_{2}(\epsilon) & \leq \frac{1}{\sqrt{\epsilon}} \sum_{n=\left[\frac{1}{\epsilon^{2}}\right]+1}^{\infty} \frac{\eta}{n^{5 / 4} g(\sqrt{n})} \\
& \leq \sum_{n=\left[\frac{1}{\epsilon^{2}}\right]+1}^{\infty} \frac{\eta}{n g(\sqrt{n})}=o(1) f_{1}(\epsilon) .
\end{aligned}
$$


By (2.9)-(2.11), note that $T_{g}\left(\frac{1}{\sqrt{\epsilon}}\right)=o(1)$, as $\epsilon \rightarrow 0$, we have

$$
\epsilon^{2} \lambda(\epsilon)-\sigma^{2}=o(1)\left(h_{1}(\epsilon)+f_{1}(\epsilon)\right), \quad \text { as } \epsilon \rightarrow 0
$$

This completes the proof of Theorem 1.2.

Remark 2.1 If $g(x)=|x|^{\delta}, 0<\delta<1$, then $f_{1}(\epsilon)=O\left(\epsilon^{\delta}\right), h_{1}(\epsilon)=O\left(\epsilon^{\delta}\right)$. By Theorem 1.2, we get

$$
\epsilon^{2} \lambda(\epsilon)-\sigma^{2}=o\left(\epsilon^{\delta}\right), \quad \text { as } \epsilon \rightarrow 0
$$

Remark 2.2 If $g(x)=|x|, \delta=1$, then $\frac{1}{\sqrt{\epsilon}} f_{2}(\epsilon)=O(\epsilon), f_{1}(\epsilon)=O(\epsilon), h_{1}(\epsilon)=O(\epsilon), h_{2}(\epsilon)=O(\epsilon)$. By (2.9), we get

$$
\epsilon^{2} \lambda(\epsilon)-\sigma^{2}=O(\epsilon), \quad \text { as } \epsilon \rightarrow 0 \text {. }
$$

\section{Competing interests}

The authors declare that they have no competing interests.

\section{Authors' contributions}

All authors read and approved the final manuscript.

\section{Acknowledgements}

The authors are very grateful to the referees and editors for their valuable comments and some helpful suggestions that improved the clarity and readability of the paper.

\section{Received: 27 May 2012 Accepted: 20 August 2012 Published: 4 September 2012}

\section{References}

1. Heyde, CC: A supplement to the strong law of large numbers. J. Appl. Probab. 12, 903-907 (1975)

2. Chen, R: A remark on the strong law of large numbers. Proc. Am. Math. Soc. 61, 112-116 (1976)

3. Gut, A, Spǎtaru, A: Precise asymptotics in the Baum-Kate and Davis law of large numbers. J. Math. Anal. Appl. 248, 233-246 (2000)

4. Lanzinger, H, Stadtmüller, U: Refined Baum-Katz laws for weighted sums of iid random variables. Stat. Probab. Lett. 69, 357-368 (2004)

5. Liu, WD, Lin, ZY: Precise asymptotic for a new kind of complete moment convergence. Stat. Probab. Lett. 76, 1787-1799 (2006)

6. Klesov, Ol: On the convergence rate in a theorem of Heyde. Theory Probab. Math. Stat. 49, $83-87$ (1994)

7. He, JJ, Xie, TF: Asymptotic property for some series of probability. Acta Math. Appl. Sin. (2012). doi:10.1007/s10255-012-0138-6

8. Gut, A, Steinebach, J: Convergence rates in Precise asymptotics. J. Math. Anal. Appl. 390, 1-14 (2012)

9. Bikelis, A: Estimates of the remainder in the central limit theorem. Litovsk. Mat. Sb. 6, 323-346 (1966)

doi:10.1186/1029-242X-2012-195

Cite this article as: He and Xie: A supplement to the convergence rate in a theorem of Heyde. Journal of Inequalities and Applications 2012 2012:195. 